\title{
HERMITIAN FUNCTIONALS ON $B$-ALGEBRAS AND DUALITY CHARACTERIZATIONS OF $C^{*}$-ALGEBRAS
}

\author{
BY \\ ROBERT T. MOORE( $\left.{ }^{1}\right)$
}

\begin{abstract}
The hermitian functionals on a unital complex Banach algebra are defined here to be those in the real span of the normalized states (tangent functionals to the unit ball at the identity). It is shown that every functional $f$ in the dual $A^{\prime}$ of $A$ can be decomposed as $f=h+i k$, where $h$ and $k$ are hermitian functionals. Moreover, this decomposition is unique for every $f \in A^{\prime}$ iff $A$ admits an involution making it a $C^{*}$-algebra, and then the hermitian functionals reduce to the usual real or symmetric functionals. A second characterization of $C^{*}$-algebras is given in terms of the separation properties of the hermitian elements of $A$ (real numerical range) as functionals on $A^{\prime}$. The possibility of analogous theorems for vector states and matrix element functionals on operator algebras is discussed, and potential applications to the representation theory of locally compact groups are illustrated.
\end{abstract}

Introduction. As the abstract indicates, we introduce here the notion of a hermitian functional on an arbitrary complex Banach algebra ( $B$-algebra) with norm-one unit; these functionals generalize real measures on a compact Hausdorff space (qua functionals on $C(X)$ ) and the real or symmetric functionals on a unital $C^{*}$-algebra. Our definition exploits the earlier involution-independent geometrical characterization of the positive functionals or states on a $C^{*}$-algebra, due to Bohnenblust and Karlin [2], as well as Lumer's strikingly successful use of this to define states on general $B$-algebras and hermitian members of such algebras [7]. We show rather easily here that the dual space $A^{\prime}$ of $A$ is the complex span of the states (a mild improvement of the Bohnenblust-Karlin vertex theorem [2]) and thus that every $f \in A^{\prime}$ can be expressed as $f=h+i k$ for suitable "real"and "imaginary" hermitian parts $h$ and $k$. The entire dual space of $A$ is thus brought into play in the geometrical study of $A$, making possible two considerably deeper duality characterizations of $C^{*}$-algebras: $A$ admits a $C^{*}$ involution iff $A^{\prime}$ decomposes as a real direct sum $A^{\prime}=H\left(A^{\prime}\right)+i H\left(A^{\prime}\right)$ of hermitians, or equivalently iff the hermitians in $A$ (or their complex span) separate points in $A^{\prime}$. (This brings the study

Received by the editors March 20, 1970 and, in revised form, December 15, 1970.

AMS 1969 subject classifications. Primary 4650, 4660; Secondary 4665, 2260.

$K e y$ words and phrases. States on $B$-algebras, positive functionals on $B$-algebras, hermitian functionals on $B$-algebras, symmetric functionals on $B$-algebras, real functionals on $B$-algebras, duality theory of $B$-algebras, characterizations of $C^{*}$-algebras, bidual $B$-algebras with Arens multiplication, weak topologies on $B$-algebras, continuous group representations in $B$-algebras, point states on operator algebras, weak-operator topology.

(1) The author gratefully acknowledges partial support of this research by the National Science Foundation through NSF GP 12548. 
of $B$ - and $C^{*}$-algebras more fully within the scope of the program of duality theory, which seeks to reduce all important properties of a locally convex space or algebra to properties of its dual space.) Both of these results rely heavily upon the rather deep geometrical characterization of $C^{*}$-algebras first given by Vidav [10] and progressively refined by Lumer [7], Berkson [1], Glickfeld [6] and Palmer [8]. The proof of the first also employs the natural generalization to $B$-algebras of the enveloping von Neumann (or $W^{*}$-) algebra of a $C^{*}$-algebra, as developed in the recent notes of Bonsall and Duncan [3]: the second dual $A^{\prime \prime}$ of $A$, as a unital $B$-algebra with Arens multiplication, is first shown to be $C^{*}$, and then the canonical image of $A$ in $A^{\prime \prime}$ is shown to be a selfadjoint subalgebra. (Thus $A^{\prime \prime}$ is in fact the enveloping $W^{*}$-algebra.)

A few very preliminary results in an analogous investigation of vector states and matrix element functionals on operator algebras are mentioned in $\S 4$, along with some open questions and some primitive-but-suggestive examples of potential applications to group representations.

2. Formulation of the principal theorems. The decomposition of $A^{\prime}$ obtained below is motivated by a much simpler decomposition which occurs in the dual of a complex unital $B$-algebra with continuous involution *. (These decompositions coincide if and only if $A$ is $C^{*}$.) We recall that every $f \in A^{\prime}$ has an adjoint $f^{*} \in A^{\prime}$ defined by the formula

$$
f^{*}(a)=f\left(a^{*}\right)^{*}
$$

for all $a \in A$. (Here we use $*$ also for complex conjugation in $C$.) Then the standard expression

$$
f=2^{-1}\left(f+f^{*}\right)+i(2 i)^{-1}\left(f-f^{*}\right)=\operatorname{Re}(f)+i \operatorname{Im}(f)
$$

decomposes every $f \in A^{\prime}$ uniquely as a sum of a symmetric functional $\left(\operatorname{Re}(f)^{*}=\operatorname{Re}(f)\right)$ and a skew-symmetric one $\left(\operatorname{Im}(f)^{*}=\operatorname{Im}(f)\right.$ so $(i \operatorname{Im}(f))^{*}$ $=-(i \operatorname{Im}(f)))$. If $S\left(A^{\prime}\right)=\left\{g \in A^{\prime}: g^{*}=g\right\}$, then we have

$$
A^{\prime}=S\left(A^{\prime}\right) \oplus i S\left(A^{\prime}\right)
$$

as a direct sum of closed real $B$-subspaces.

Now, in the special case where $A$ is $C^{*}$, the symmetric functionals admit a decomposition into positive and negative parts. Specifically, if the normalized positive functionals are

$$
P\left(A^{\prime}\right)=\left\{\omega \in A^{\prime}: \omega\left(a a^{*}\right) \geqq 0 \text { for all } a \in A \text { and } \omega(I)=1\right\}
$$

then for each $s \in S\left(A^{\prime}\right)$ there exist scalars $\alpha_{1}, \alpha_{2} \geqq 0$ and positive functionals $\omega_{1}, \omega_{2} \in P\left(A^{\prime}\right)$ such that

$$
s=\alpha_{1} \omega_{1}-\alpha_{2} \omega_{2}
$$


(The existence of such a decomposition is given in Corollary 2.6.4 of Dixmier [5], and uniqueness subject to a disjointness condition is established in Corollary 12.3.4 there.) But Bohnenblust and Karlin [2] have given a description of $P\left(A^{\prime}\right)$ entirely independent of *:

$$
P\left(A^{\prime}\right)=\left\{\omega \in A^{\prime}: \omega(I)=1=\|\omega\|^{\prime}\right\}
$$

and Lumer [7] has used the generalization of this to define an analogous weak*-compact convex space of (normalized) states for an arbitrary complex unital $B$-algebra:

$$
\Omega\left(A^{\prime}\right)=\left\{\omega \in A^{\prime}: \omega(I)=1=\|\omega\|^{\prime}\right\} .
$$

In the same spirit, we take (5) as the definition of the hermitian functionals on $A$

$$
\begin{aligned}
H\left(A^{\prime}\right) & =\left\{\alpha_{1} \omega_{1}-\alpha_{2} \omega_{2}: \alpha_{1}, \alpha_{2} \geqq 0, \omega_{1}, \omega_{2} \in \Omega\left(A^{\prime}\right)\right\} \\
& =\text { real span } \Omega\left(A^{\prime}\right) .
\end{aligned}
$$

Just as Lumer's states retain most of the useful properties of the positive functionals on a $C^{*}$-algebra, our hermitian functionals retain most of those of the symmetric functionals. The most important property that persists is a weakened form of (3).

THEOREM 1. Let $A$ be a complex unital B-algebra.

(a) The hermitian functionals $H\left(A^{\prime}\right)$ form a real subspace of the dual $A^{\prime}$ of $A$, $H\left(A^{\prime}\right)$ is a real B-space with respect to the natural hermitian norm for $h \in H\left(A^{\prime}\right)$ :

$$
\|h\|_{H}=\inf \left\{\alpha+\beta: h=\alpha \omega_{1}-\beta \omega_{2} \text { for } \alpha, \beta \geqq 0 \text { and } \omega_{1}, \omega_{2} \in \Omega\left(A^{\prime}\right)\right\},
$$

and the natural injection of $\left(H\left(A^{\prime}\right),\|\cdot\|_{H}\right)$ into $\left(A^{\prime},\|\cdot\|^{\prime}\right)$ is continuous.

$$
A^{\prime}=H\left(A^{\prime}\right)+i H\left(A^{\prime}\right)=\text { complex } \operatorname{span}\left(\Omega\left(A^{\prime}\right)\right) \text {. }
$$

Also

(b) Moreover, $A$ admits a $C^{*}$ involution $\left({ }^{2}\right)$ iff

$$
N\left(A^{\prime}\right)=H\left(A^{\prime}\right) \cap i H\left(A^{\prime}\right)=\{0\} .
$$

In that case, the sum in (9) is direct and $H\left(A^{\prime}\right)=S\left(A^{\prime}\right)$ so that (9) reduces to (3), and $H\left(A^{\prime}\right)$ is a closed subspace of $A^{\prime}$.

Remarks. (1) The claim in (9) that the subspace $K=$ complex span $\left(\Omega\left(A^{\prime}\right)\right)$ exhausts $A^{\prime}$ can be viewed as a useful strengthening of the Bohnenblust-Karlin vertex theorem [2]. The latter asserts that $\Omega\left(A^{\prime}\right)$ separates points in $A$, and is easily seen to be equivalent to the claim that $K$ separates points in $A^{\prime}$. By a duality argument (which also appears in the proof of Theorem 2) one sees that $K$ separates points iff it is weak-* dense in $A^{\prime}$, and the first claim in Theorem 1 asserts that it actually coincides with $A^{\prime}$.

$\left({ }^{2}\right)$ That is, $A$ admits an involution * such that it is isometrically *-isomorphic to an adjointclosed subalgebra of the operator algebra $B(H)$ on some Hilbert space $H$. 
(2) It is natural to ask when the "natural" norm on $H\left(A^{\prime}\right)$ mentioned in (a) is topologically equivalent to the relative dual norm on $H\left(A^{\prime}\right)$ inherited from $A^{\prime}$. A routine application of the open mapping theorem shows that this occurs iff $H\left(A^{\prime}\right)$ is a closed real subspace of $A^{\prime}$ (hence a real $B$-space with respect to the dual norm). This is true, for example, in (b) above, when the sum $A^{\prime}=H\left(A^{\prime}\right) \oplus i H\left(A^{\prime}\right)$ is algebraically direct. Methods communicated to me by $\mathrm{F}$. F. Bonsall can be used to show that $H\left(A^{\prime}\right)$ need not be closed, so that then the natural norm gives $H\left(A^{\prime}\right)$ a strictly finer topology; this occurs in the disc algebra $A(D)$ of functions analytic in the interior of the unit disc and continuous on the boundary. (Details will be published elsewhere.)

The thrust of Theorem 1 can be summarized in the observation: every $B$-algebra has enough hermitian functionals, and failure to be $C^{*}$ is associated with the presence of too many such functionals (those in $N\left(A^{\prime}\right)$ ). The appearance of these "nonstandard" hermitians can be attributed in part to the scarcity of hermitian elements in $A$. We recall that by Lumer [7] we may define the hermitians in $A$ as follows

$$
H(A)=\left\{a \in A: \omega(a) \in \boldsymbol{R} \text { for all } \omega \in \Omega\left(A^{\prime}\right)\right\} .
$$

THEOREM 2. Let $A$ be a complex unital B-algebra. Then the following are equivalent:

(i) The hermitians $H(A)$ separate the states in $\Omega\left(A^{\prime}\right)$.

(ii) The hermitians $H(A)$ separate points in $A^{\prime}$.

(iii) There exists an involution * on $A$ with respect to which $A$ is a $C^{*}$-algebra.

ACKNOWLEDGements. I wish to thank F. F. Bonsall for calling to my attention an important error (fortunately, a repairable one) in the first draft of this article. I would also like to thank A. M. Sinclair for suggesting the importance of the number $\|h\|_{H}=\inf \left\{\alpha+\beta: h=\alpha_{1} \omega_{1}-\alpha_{2} \omega_{2}\right.$, etc. $\}$ which coincides with the dual norm $\|h\|^{\prime}$ if $A$ is $C^{*}$. This number is in fact equal to a much clumsier and less natural "coset norm" which I had previously employed here. (Both appear in the proof. See below.) Sinclair has also independently proved (9), using an interesting argument which employs no measure theory, being based purely upon convexity and separation methods.

3. Proof of the main theorem. The proof of Theorem 1 turns upon two rather easy lemmas which may be of some independent interest. The second claim also requires an application of the deep theorem of Vidav-Palmer to the second dual $A^{\prime \prime}$ of $A$ when the latter is treated as a unital $B$-algebra with the Arens multiplication. This machinery is available in precisely the right form in the excellent notes of Bonsall and Duncan [3] on numerical range, and the reader will be assumed to be familiar with that treatment. (Indeed, the method of proof used here was suggested to me by the treatment of $A^{\prime \prime}$ in $\S 12$ of these notes; I am indebted to the authors for the privilege of seeing a preprint.) 
For the first lemma, we recall that

$$
W(a)=\left\{\omega(a): \omega \in \Omega\left(A^{\prime}\right)\right\}
$$

is the numerical range of $a \in A$, and its numerical radius is

$$
w(a)=\sup \{|\lambda|: \lambda \in W(a)\} .
$$

The latter is a norm on $A$ topologically equivalent to the given norm via

$$
w(a) \leqq\|a\| \leqq e w(a)
$$

(cf. [2]) so that, with primes denoting dual norms on $A^{\prime}$,

$$
w^{\prime}(f) \geqq\|f\|^{\prime} \geqq e^{-1} w^{\prime}(f)
$$

is an equivalent norm for $A^{\prime}$. We then isolate the tangent functionals to the $w$-unit ball at $I$, or the " $w$-states"

$$
\Omega_{w}\left(A^{\prime}\right)=\left\{\omega \in A^{\prime}: \omega(I)=1=w^{\prime}(\omega)\right\} .
$$

LEMma A. (a) The $w$-states coincide with the states

$$
\Omega_{w}\left(A^{\prime}\right)=\Omega\left(A^{\prime}\right) .
$$

(b) In particular, a functional $f \in A^{\prime}$ is a state iff it maps every $a \in A$ into its numerical range

$$
\Omega\left(A^{\prime}\right)=\left\{f \in A^{\prime}: f(a) \in W(a) \text { for all } a \in A\right\} .
$$

Remark. The characterization (14) is analogous to the much deeper result of Gleason-Kahane-Zelasko (cf. [3]) describing the multiplicative functionals on $A$ : $f$ is multiplicative iff it maps every $a \in A$ into its spectrum.

Proof. (a) The first inequality in (12) shows that if $\omega \in \Omega_{w}\left(A^{\prime}\right)$, then $\|\omega\|^{\prime} \leqq w^{\prime}(\omega)$ $=1$, and since $\omega(I)=1,\|\omega\|^{\prime}=1$ and $\omega \in \Omega\left(A^{\prime}\right)$. For the converse (and for (b)), we notice that if $f(a) \in W(a)$ for all $a \in A$ (necessarily true if $f \in \Omega\left(A^{\prime}\right)$ ) then $|f(a)|$ $\leqq w(a)$ for all $a \in A$ and $w^{\prime}(f) \leqq 1$. But since $w(I)=\{1\}$ by inspection, any such $f$ has $f(I)=1$ and $w^{\prime}(f)=1$, so $f \in \Omega_{w}\left(A^{\prime}\right)$. Thus (13) is proved.

To complete (b), what was said above now implies that the left-hand side of (14) contains the right, and the reverse inclusion holds by definition.

The second lemma is required in the proof of (b); it is unlikely to be new but I have been unable to locate a reference.

Lemma B. Let $A$ be a closed unital subalgebra of a $C^{*}$-algebra $C$. Then $A$ is $a$ $C^{*}$-subalgebra (i.e. closed with respect to ${ }^{*}$ ) iff for every $f=\operatorname{Re}(f)+i \operatorname{Im}(f) \in C^{\prime}$ which vanishes on $A, \operatorname{Re}(f)$ and $\operatorname{Im}(f)$ vanish on $A$ as well.

Proof. We recall that $\operatorname{Re}(f)$ and $\operatorname{Im}(f)$ are the unique hermitian functionals on $C$ which are defined on the hermitians $H(C)$ in $C$ by $\operatorname{Re}(f)(b)=\operatorname{Re}(f(b)), \operatorname{Im}(f)(b)$ $=\operatorname{Im}(f(b))$ for all $b \in H(C)$. 
Thus if $A$ is $C^{*}$, the usual calculation $a=2^{-1}\left(a+a^{*}\right)+(2 i)^{-1}\left(a-a^{*}\right)$ shows that $A=H(A) \oplus i H(A)$ for $H(A)$ the set of hermitians in $A$. If $f$ vanishes on $A$, then $f$, $\operatorname{Re}(f)$, and $\operatorname{Im}(f)$ all vanish on $H(A)$. But then if $a=b+i c$ for $b, c$ in $H(A)$, $\operatorname{Re}(f)(a)=\operatorname{Re}(f)(b)+i \operatorname{Re}(f)(c)=0$, and $\operatorname{Im}(f)(a)=0$ similarly, so $\operatorname{Re}(f)$ and $\operatorname{Im}(f)$ vanish on $A$.

On the other hand, if $A$ is not a $C^{*}$-subalgebra, there must be an $a \in A$ with $a^{*} \notin A$, and by the Hahn-Banach theorem, there is an $f \in C^{\prime}$ which vanishes on $A$ and is nonzero at $a^{*}$ (say $f\left(a^{*}\right)=1$ ). Thus at least one of the hermitian functionals $\operatorname{Re}(f)$ and $\operatorname{Im}(f)$ must be nonzero at $a^{*}$. But for every hermitian functional $h \in H\left(C^{\prime}\right)$ and every $a \in C$ it is true that $h(a)=h\left(a^{*}\right)^{*}$, so at least one of $\operatorname{Re}(f)$ and $\operatorname{Im}(f)$ fails to vanish on $a$ as well. By contraposition, the converse is proved.

Proof of Theorem 1(a). The idea of our proof is close in spirit to the one frequently used (e.g. in [5]) to decompose $S\left(A^{\prime}\right)$ with respect to $P\left(A^{\prime}\right)$ when $A$ is $C^{*}$ : we identify $A$ with functions on the weak-*-compact convex set $\Omega\left(A^{\prime}\right)$, and $A^{\prime}$ with suitable measures there. Specifically, if $a \in A$ the formula $\hat{a}(\omega)=\omega(a)$ defines a continuous function $\hat{a} \in C\left(\Omega\left(A^{\prime}\right), C\right)$, and it is clear that $w(a)=\|\hat{a}\|_{\infty}=\sup \left\{|\hat{a}(\omega)|: \omega \in \Omega\left(A^{\prime}\right)\right\}$. The function $I$ is identically 1 .

The algebraic claim in Theorem 1(a) is established by means of the HahnBanach and Riesz theorems, which guarantee that for each $f \in A^{\prime}$ there exists at least one Borel measure $\mu_{f}$ such that for all $a \in A$

$$
f(a)=\int_{\Omega\left(A^{\prime}\right)} \hat{a}(\omega) d \mu_{f}(\omega)
$$

By the usual decomposition of measures

$$
\mu_{f}=\sum\left\{i^{j} \alpha_{j} \pi_{j}: 0 \leqq j \leqq 3\right\}
$$

where $\alpha_{j} \geqq 0$ and $\pi_{j}$ is a probability measure on $\Omega\left(A^{\prime}\right)$. But if $\pi$ is a probability measure, the functional $\omega_{\pi}(a)=\int_{\Omega\left(A^{\prime}\right)} \hat{a}(\omega) d \pi(\omega)$ satisfies $\omega_{\pi}(I)=1$ and $\left|\omega_{\pi}(a)\right|$ $\leqq\|\hat{a}\|_{\infty}=w(a)$, so $\omega_{\pi} \in \Omega_{w}\left(A^{\prime}\right)$. Thus by Lemma $\mathrm{A}$, there are states $\omega_{j} \in \Omega\left(A^{\prime}\right)$ $=\Omega_{w}\left(A^{\prime}\right)$ corresponding to the $\pi_{j}$ in (8), and for each $a \in A$

$$
f(a)=\sum\left\{i^{j} \alpha_{j} \int_{\Omega\left(A^{\prime}\right)} \hat{a}(\omega) d \pi_{j}(\omega)\right\}=\sum i^{j} \alpha_{j} \omega_{j}(a) .
$$

Consequently

$$
\begin{aligned}
f= & \left(\alpha_{1} \omega_{1}-\alpha_{3} \omega_{3}\right)+i\left(\alpha_{2} \omega_{2}-\alpha_{4} \omega_{4}\right) \in H\left(A^{\prime}\right)+i H\left(A^{\prime}\right) \\
& \subset \text { complex } \operatorname{span}\left(\Omega\left(A^{\prime}\right)\right)
\end{aligned}
$$

and equation (9) in Theorem 1(a) follows.

To establish the topological claim, let us identify $A$ (normed by $w$ ) isometrically as a subspace of $C=C\left(\Omega\left(A^{\prime}\right), C\right)$. We check that the restriction map $\rho: C^{\prime} \rightarrow A^{\prime}$, from the dual $C^{\prime}$ of $C$ (measures on $\Omega\left(A^{\prime}\right)$ ) onto the dual of $A$, carries the space $R$ of real measures onto $H\left(A^{\prime}\right)$. We also show that $\rho(R)$-alias-H(A') is isomorphic as 
a vector space to $R /\left(R \cap A^{\perp}\right)$, and that the latter is a real $B$-space, so that its $B$-space structure can be transported to $H\left(A^{\prime}\right)$. (Here $A^{\perp}=\left\{f \in C^{\prime}: f(A)=\{0\}\right\}$.)

Since every real measure $\mu$ can be written in the form $\mu=\alpha_{1} \pi_{1}-\alpha_{2} \pi_{2}$, for $\alpha_{i} \geqq 0$ and $\pi_{i}$ a probability measure on $\Omega\left(A^{\prime}\right)(i=1,2)$, the argument based upon Lemma A used above shows that the $\pi_{i}$ restrict to states $\omega_{i}$ on $A$, and linearity of restriction implies that $\mu$ restricts to a member of $H\left(A^{\prime}\right)$. Thus $\rho(R) \subset H\left(A^{\prime}\right)$. But if $h=\alpha_{1} \omega_{1}-\alpha_{3} \omega_{3} \in H\left(A^{\prime}\right)$, any Hahn-Banach extensions $\pi_{i}$ of the $\omega_{i}$ must satisfy $\pi_{i}(I)=\omega_{i}(I)=1=w^{\prime}\left(\omega_{i}\right)=\left\|\omega_{i}\right\|_{\infty}^{\prime}=\left\|\pi_{i}\right\|_{\infty}^{\prime}$, whence the $\pi_{i}$ are (represented by) probability measures and $\mu=\alpha_{1} \pi_{1}-\alpha_{3} \pi_{3} \in R$ with $\rho(\mu)=h$.

Next, since $A^{\perp}=\operatorname{ker}(\rho), A^{\prime} \cong C^{\prime} / A^{\perp}$ as vector spaces, and a standard folk-lemma asserts that this isomorphism is an isometry between $w^{\prime}$ on $A^{\prime}$ and the quotient norm $\left\|\mu+A^{\perp}\right\|_{q}=\inf \left\{\|\mu+\nu\|_{\infty}^{\prime}: \nu \in A^{\perp}\right\}$ on $C^{\prime} / A^{\perp}$. (Every $\mu+\nu$ restricts to a functional of smaller norm on $A$, so that the norm $w^{\prime}(\rho(\mu))$ of the restriction is dominated by $\left\|\mu+A^{\perp}\right\|_{q}$, while every $\rho(\mu)$ has at least one Hahn-Banach extension $\sigma \in \mu+A^{\perp}$ with $\|\sigma\|_{\infty}^{\prime}=w^{\prime}(\rho(\mu))$, which proves equality.) Consequently, $H\left(A^{\prime}\right)$ with $w^{\prime}$ as norm can be identified with $\rho(R)$ equipped with the quotient norm $\|\cdot\|_{q}$.

Similarly, the restriction $\left.\rho\right|_{R}$ of $\rho$ to $R$ has kernel $R \cap A^{\perp}$, so that $\rho(R)$ is also isomorphic to $R /\left(R \cap A^{\perp}\right)$. Since both $R$ and $A^{\perp}$ are closed in $C^{\prime}$ (hence they are real $B$-spaces), $R \cap A^{\perp}$ is closed and the quotient $R /\left(R \cap A^{\perp}\right)$ is a real $B$-space when equipped with the quotient norm $\left\|\mu+R \cap A^{\perp}\right\|_{q}=\inf \left\{\|\mu+\nu\|_{\infty}: \nu \in R \cap A^{\perp}\right\}$. The various identifications finally identify $\mu+A^{\perp}$ with $\mu+R \cap A^{\perp}$ when $\mu \in R$, and $\left\|\mu+A^{\perp}\right\|_{q} \leqq\left\|\mu+R \cap A^{\perp}\right\|_{q}$ by inspection, whence by our last paragraph the map of $\left(R /\left(R \cup A^{\perp}\right),\|\cdot\|_{q}\right)$ onto $\left(H\left(A^{\prime}\right), w^{\prime}\right)$ is norm-decreasing and is continuous into $A^{\prime}$ as claimed.

Finally, we check that if $h \in H\left(A^{\prime}\right)$ is the hermitian functional whose real representing measures are described by the coset $\mu+R \cap A^{\perp}$ ( $\mu$ real), then the quotient norm $\left\|\mu+R \cap A^{\perp}\right\|_{q}$ coincides with the hermitian norm $\|h\|_{H}$ $=\inf \left\{\alpha+\beta: h=\alpha \omega_{1}-\beta \omega_{2}\right.$ for $\left.\alpha, \beta \geqq 0, \omega_{1}, \omega_{2} \in \Omega\left(A^{\prime}\right)\right\}$. First, if $\mu+\nu$ represents $h$ (i.e. $h(a)=\int_{\Omega\left(A^{\prime}\right)} \hat{a}(\omega) d(\mu+\nu)$ ) we can calculate its total variation $\|\mu+\nu\|_{\infty}^{\prime}$ by its Jordan decomposition $\mu+\nu=\alpha \pi_{1}-\beta \pi_{2}$ with the $\pi_{i}$ probability measures on $\Omega\left(A^{\prime}\right), \alpha, \beta \geqq 0$, and $\|\mu+\nu\|_{\infty}^{\prime}=\alpha+\beta$. But then the $\pi_{i}$ restrict to states $\omega_{i}$ and $h$ $=\alpha \omega_{1}-\beta \omega_{2}$ as before, so $\|h\|_{H} \leqq \alpha+\beta=\|\mu+\nu\|_{\infty}^{\prime}$ and we conclude that $\|h\|_{H}$ $\leqq \inf \left\{\|\mu+\nu\|_{\infty}^{\prime}: \nu \in R \cap A^{\perp}\right\}=\|\mu+R \cap A\|_{q}$. On the other hand, if $h=\alpha \omega_{1}-\beta \omega_{2}$ for $\omega_{1}, \omega_{2} \in \Omega\left(A^{\prime}\right)$, the $\omega_{i}$ extend to probability measures $\pi_{i}$ and the real measure $\alpha \pi_{1}-\beta \pi_{2}$ represents $h$ and must be of the form $\mu+\nu$ for $\nu \in R \cap A^{\perp}$. But then, by standard measure theory, the total variations satisfy $\|\mu+\nu\|_{\infty}^{\prime} \leqq\left\|\alpha \pi_{1}\right\|_{\infty}^{\prime}+\left\|\beta \pi_{2}\right\|_{\infty}^{\prime}$ $=\alpha+\beta$. This suffices to prove the reverse inequality, so that $\|h\|_{H}=\left\|\mu+R \cap A^{\perp}\right\|_{q}$ as claimed.

Proof of Theorem 1(b). The idea of this part of the proof is to use the decomposition of $A^{\prime}$,

$$
A^{\prime}=H\left(A^{\prime}\right) \oplus i H\left(A^{\prime}\right),
$$


to decompose the second dual as

$$
A^{\prime \prime}=H\left(A^{\prime \prime}\right) \oplus i H\left(A^{\prime \prime}\right)
$$

where $H\left(A^{\prime \prime}\right)$ is exactly the set of hermitian elements of the unital $B$-algebra $A^{\prime \prime}$ obtained from the Arens multiplication. Once this is established, the VidavPalmer theorem [8] implies that $A^{\prime \prime}$ is a $C^{*}$-algebra, and the only remaining step is to check that the canonical image of $A$ in $A^{\prime \prime}$ is $C^{*}$, which we do by Lemma $\mathrm{B}$.

The decomposition of $A^{\prime \prime}$ is obtained by defining the obvious involution * on $A^{\prime}$ and then repeating the steps (1)-(3) at the beginning of $\S 2$ on $A^{\prime \prime}$ rather than on $A^{\prime}$. Specifically, if $f=h+i k$ (for $h, k$ in $H\left(A^{\prime}\right)$ ) is the unique representation of $f \in A^{\prime}$, then

$$
f^{*}=h-i k
$$

defines the desired involution uniquely. It is evident that $f^{* *}=f$, but we must check that ${ }^{*}$ is continuous and complex conjugate-linear. Let $E_{r}$ and $E_{i}$ be the disjoint real-linear projections onto $H\left(A^{\prime}\right)$ and $i H\left(A^{\prime}\right)$ respectively. Then clearly

$$
f^{*}=\left(E_{r}-E_{i}\right) f
$$

which immediately implies real-linearity of *. Complex conjugate-linearity follows easily then from the fact that $H\left(A^{\prime}\right)$ and $i H\left(A^{\prime}\right)$ are real subspaces, and from uniqueness of decomposition, by the calculation

$$
\begin{aligned}
{\left[e^{i \theta}(h+i k)\right]^{*} } & =[(\cos (\theta) h-\sin (\theta) k)+i(\sin (\theta) h+\cos (\theta) k)]^{*} \\
& =(\cos (\theta) h-\sin (\theta) k)-i(\sin (\theta) h+\cos (\theta) k) \\
& =e^{-i \theta}(h-i k)=\left(e^{i \theta}\right)^{*}[h+i k]^{*} .
\end{aligned}
$$

The continuity claim is deeper, but reduces quickly by (14) to the claim that $E_{r}$ and $E_{i}$ are continuous, and since multiplication by $i$ is a homeomorphism and projections are continuous iff their kernels are closed (by the closed graph theorem) it suffices to check that $H\left(A^{\prime}\right)$ is norm-closed in $A^{\prime}$. In the spirit of Remark 2 following the statement of the theorem, we check that the dual norm topology of $H\left(A^{\prime}\right)$ is equivalent to the $B$-space topology it obtains from the natural norm in (a), which we denote by $\|\cdot\|_{H}$ here. Transporting $\|\cdot\|_{H}$ to $i H\left(A^{\prime}\right)$, we may equip $A^{\prime}=H\left(A^{\prime}\right) \oplus i H\left(A^{\prime}\right)$ with the direct sum topology derived from $\|\cdot\|_{H}$ to obtain a $B$-space topology which is easily checked to be finer than the dual norm topology on $A^{\prime}$. Applying the open mapping theorem to the identification, it becomes a topological isomorphism which identifies the two topologies on $H\left(A^{\prime}\right)$. Closure of $H\left(A^{\prime}\right)$ then follows from $\|\cdot\|_{H}$-completeness.

Then the involution is lifted over to $F \in A^{\prime \prime}$ by

$$
F^{*}(f)=F\left(f^{*}\right)^{*}
$$

so that if $S\left(A^{\prime \prime}\right)=\left\{F \in A^{\prime \prime}: F^{*}=F\right\}$, we have as in (3) of $\S 2$ the algebraic decomposition

$$
A^{\prime \prime}=S\left(A^{\prime \prime}\right)+i S\left(A^{\prime \prime}\right)
$$


But each $F \in S\left(A^{\prime \prime}\right)$ maps $\Omega\left(A^{\prime}\right)$ into $R$, since each $\omega \in \Omega\left(A^{\prime}\right)$ has $\omega^{*}=\omega$ $\left(=\left(E_{r}-E_{i}\right) \omega\right)$ and

$$
F(\omega)=F^{*}(\omega)=F\left(\omega^{*}\right)^{*}=F(\omega)^{*} .
$$

Consequently, Corollary 12.3 in Bonsall-Duncan [3] guarantees that each such $F \in S\left(A^{\prime \prime}\right)$ is in the hermitians $H\left(A^{\prime \prime}\right)$ when $A^{\prime \prime}$ is treated as a unital $B$-algebra, and it is certainly true then that (12) holds as desired.

Finally, since $A^{\prime \prime}=C$ is now known to be a $C^{*}$-algebra, and since the canonical injection of $A$ into $A^{\prime \prime}$ is an algebra isomorphism ( $\$ 12$ of [3]) we may apply Lemma B. Thus if $A$ failed to be a *-subalgebra, we could find $f \in C^{\prime}$ vanishing on $A$ such that the symmetric parts $\operatorname{Re}(f)$ and $\operatorname{Im}(f)$ do not both vanish. As discussed in $\S 2$, symmetric functionals $h$ are hermitian and $h=\alpha_{1} \omega_{1}-\alpha_{2} \omega_{2}$, for the $\omega_{i}$ states of $C$. Then since states of $C$ clearly restrict to states of $A, \operatorname{Re}(f)$ and $\operatorname{Im}(f)$ restrict to hermitians on $A-$ call them $h$ and $k$. But since $h+i k$ vanishes on $A, h=-i k$ would be a nontrivial member of $N\left(A^{\prime}\right)$, which is impossible. Hence $A$ is a *-subalgebra and (b) is proved.

3. Two proofs of Theorem 2. There are two rather different lines of argument which can be used to prove Theorem 2 . The shortest uses both parts of Theorem 1 , with very little added machinery. The other uses merely the first part of Theorem 1 and a duality argument. Both require parts of the following lemma.

Lemma C. Let $A$ be a complex unital B-algebra.

(a) Every nontrivial hermitian functional $h \in H\left(A^{\prime}\right)$ which vanishes on $H(A)$ has the form

$$
h=\alpha\left(\omega_{1}-\omega_{2}\right), \quad \alpha>0, \omega_{1} \neq \omega_{2},
$$

where $\omega_{1}-\omega_{2}$ vanishes on $H(A)$.

(b) Every $h \in N\left(A^{\prime}\right)$ vanishes identically on $H(A)$ and, if it is nontrivial, has the form (1).

Proof. (a) First, $I \in H(A)$, so that any such $h=\alpha_{1} \omega_{1}-\alpha_{2} \omega_{2}, \alpha_{i} \geqq 0, \omega_{i} \in \Omega\left(A^{\prime}\right)$ must satisfy $0=h(I)=\alpha_{1} \omega_{1}(I)-\alpha_{2} \omega_{2}(I)=\alpha_{1}-\alpha_{2}$, and we take $\alpha=\alpha_{1}=\alpha_{2}$. Thus $h=\alpha\left(\omega_{1}-\omega_{2}\right)$, and this functional is nontrivial iff $\alpha>0$ and $\omega_{1} \neq \omega_{2}$, while $\omega_{1}-\omega_{2}$ must vanish on $H(A)$ if $h$ does.

(b) Since $\Omega\left(A^{\prime}\right)$ by definition maps $H(A)$ into $R$, so does its real span $H\left(A^{\prime}\right)$. Thus every $h \in N\left(A^{\prime}\right)=H\left(A^{\prime}\right) \cap i H\left(A^{\prime}\right)$ maps $H(A)$ into $\boldsymbol{R} \cap i \boldsymbol{R}=\{0\}$ and vanishes on $H(A)$. The rest is just (a).

Proof of Theorem 2. To prove that (i) and (ii) are equivalent, we observe first that if $H(A)$ separates points in $A^{\prime}$, it certainly separates points in $\Omega\left(A^{\prime}\right) \subset A^{\prime}$, so (ii) implies (i). Conversely, it suffices to prove that if (i) holds, every $f \in A^{\prime}$ that vanishes on $H(A)$ vanishes identically, since $A^{\prime}$ is a vector space. By Theorem 1(a), if $f \in A^{\prime}$ it can be written in at least one way as $f=h+i k$ with $h$ and $k$ in $H\left(A^{\prime}\right)$. If 
$f$ vanishes on $H(A)$, then $h$ agrees with $-i k$ there, but then since $h$ and $k$ map $H(A)$ into $\boldsymbol{R}$ (as in the proof of Lemma C), both $h$ and $-i k$ (thus $k$ ) must vanish on $H(A)$. Hence by Lemma $\mathrm{C}(\mathrm{a})$ and contraposition, both $h$ and $k$ vanish everywhere (otherwise we could find $\omega_{1} \neq \omega_{2}$ which were not separated by $H(A)$, contrary to (i)). Consequently $f$ vanishes everywhere and (i) implies (ii).

The proof will now be complete if we establish equivalence of (i) and (iii). If $H(A)$ separates points in $\Omega\left(A^{\prime}\right)$, then Lemma $\mathrm{C}(\mathrm{b})$ shows by contraposition that $N\left(A^{\prime}\right)=\{0\}$, so (iii) follows by Theorem 1(b). Conversely, if $A$ admits a $C^{*}$ involution, we know that $\Omega\left(A^{\prime}\right)=P\left(A^{\prime}\right)$ by [2], and $H(A)=\left\{a \in A: a^{*}=a\right\}$ by [7]. Since positive functionals $\omega \in P\left(A^{\prime}\right)$ on a unital $C^{*}$-algebra are real on $H(A)$, and since $A=H(A) \oplus i H(A) \quad\left(a=2^{-1}\left(a+a^{*}\right)+i(2 i)^{-1}\left(a-a^{*}\right)\right)$ it is clear that if $\omega_{1}-\omega_{2}$ vanishes on $H(A)$, it vanishes on $A$ and $\omega_{1}=\omega_{2}$. Hence $H(A)$ separates points in $P\left(A^{\prime}\right)$-alias- $\Omega\left(A^{\prime}\right)$ and (i) follows.

The second proof differs from the first only in that the implication (ii) $\Rightarrow$ (iii) replaces "(i) $\Rightarrow$ (iii)" and is proved in a different way. The following lemma contains most of the methodology.

Lemma D. Let $A$ be a complex unital B-algebra, and let $J(A)=\dot{H}(A)+i H(A)$.

(a) The subset $J(A) \subset A$ is a complex subspace which is both weakly closed and norm-closed.

(b) If $J(A)$ separates points in $A^{\prime}$, then $J(A)$ is weakly dense in $A$, hence $J(A)=A$.

Proof. (a) (Due essentially to Vidav [10].) Every $\omega$ in the separating set $\Omega\left(A^{\prime}\right)$ maps $H(A) \cap i H(A)$ into $\boldsymbol{R} \cap i \boldsymbol{R}=\{0\}$, so $H(A) \cap i H(A)=\{0\}$. Moreover, $H(A)$ is norm-closed, since a limit $a=\lim h_{\alpha}$ in $H(A)^{-}$has $\omega(a)=\lim \omega\left(h_{\alpha}\right) \in \boldsymbol{R}$ for every $\omega \in \Omega\left(A^{\prime}\right)$. Thus the projections $E_{r}$ and $E_{i}$ onto $H(A)$ and $i H(A)$ are continuous real-linear and disjoint, so $E=E_{i}+E_{r}$ is continuous real-linear and its range $J(A)$ is norm-closed real-linear. Closure under complex multiples is obvious. Since $J(A)$ is clearly convex, Mazur's theorem (Corollary 2 to II.9.2 in Schaefer [9]) insures that it is weakly closed.

(b) A subspace of any locally convex space is weakly dense iff it separates points in the dual (IV.1.3 in [9]). This is proved using the Hahn-Banach theorem in the weak topology, with the obvious fact that every weakly continuous functional is continuous: if $J$ were not weakly dense, there would exist a nontrivial $f \in A^{\prime}$ vanishing on the weak closure of $J$.

Second proof of Theorem 2. Suppose (ii) is true: $H(A)$ separates points in $A^{\prime}$. Then $J(A)$ certainly separates points, so by Lemma $\mathrm{D}(\mathrm{b}), J(A)=A$. Then by the Vidav-Palmer theorem [8], $A$ admits a $C^{*}$ involution, proving (iii).

The first paragraph of the first proof then establishes (i) $\Leftrightarrow$ (ii) $\Rightarrow$ (iii), and the implication (iii) $\Rightarrow$ (i) from the second paragraph completes the network of implications.

Remark. The fact that Theorem 2 can be proved without recourse to the $B$-algebra $A^{\prime \prime}$ raises the natural question of whether Theorem 1(b) might not admit a 
similar "intrinsic" proof. However, the present proof relies heavily upon the fact that $H\left(A^{\prime}\right)$ is closed in $A^{\prime}$; assuming that this fact is crucial, it is difficult to see how to exploit it without reference to $A^{\prime \prime}$.

4. Weak topologies on $A$ : an open problem. The following easy corollary of Theorem 1 part (a) is the primary reason for its interest, and provides the basis for most applications known to this author.

Corollary 3. The weak topology induced on $A$ by $A^{\prime}, \sigma\left(A, A^{\prime}\right)$, is exactly the coarsest vector topology on $A$ such that every $\omega \in \Omega\left(A^{\prime}\right)$ is continuous.

Proof. Since $\sigma\left(A, A^{\prime}\right)$ is the coarsest vector topology such that every $f \in A^{\prime}$ is continuous, it is finer that the " $\Omega\left(A^{\prime}\right)$ topology". But if every $\omega \in \Omega\left(A^{\prime}\right)$ is continuous with respect to some topology $\tau$ for $A$, so is every $f \in\left\{\right.$ complex span $\left.\left(\Omega\left(A^{\prime}\right)\right)\right\}$, whence by Theorem $1(\mathrm{a})$, every $f \in A^{\prime}$ is $\tau$-continuous. This proves the reverse inclusion of topologies.

As a typical application, let $\rho: G \rightarrow A$ be a homomorphism of a locally compact group $G$ into the multiplicative semigroup of a complex unital $B$-algebra $A$ such that $\rho$ carries the identity $e \in G$ into $I$. It is not difficult to prove that if the maps $g \rightarrow \omega(\rho(g)) \in C$ are continuous for every $\omega \in \Omega\left(A^{\prime}\right)$, then $g \rightarrow \rho(g)$ is normcontinuous into $A$. First, the hypothesis asserts exactly that $g \rightarrow \rho(g)$ is continuous into $A$ when the latter carries the "state topology", so it is continuous when $A$ carries the weak topology. Thus, by continuity of multiplication in $A$, every pair $(f, a) \in A^{\prime} \times A$ induces a continuous map $g \rightarrow f(\rho(g) a)$ by composition. This means that $g \rightarrow L_{g}^{\rho} \in B(a)$ defined by $L_{g}^{\rho} a=\rho(g) a$ is a weak-operator continuous representation into $B(A)$, the bounded operators on $A$. But by Theorem 2.8 in DeLeeuwGlicksberg [4], $L$ is then strongly continuous: $g \rightarrow L_{g}^{\rho} a$ is norm-continuous into $A$ for all $a \in A$. But then in particular $g \rightarrow L_{g}^{\rho} I=\rho(g) I=\rho(g)$ is norm-continuous as claimed.

In many cases of practical interest, the unital $B$-algebra $A$ which one considers is either the algebra $B(X)$ of all bounded operators on a $B$-space or perhaps a subalgebra of it. In that case, various weak-operator topologies are naturally defined on $X$. When $X$ is a Hilbert space with inner product (, ), the situation is very simple: one is led to consider the weak-operator topology on $A$, defined as the coarsest topology such that for all $u$ and $v$ in $X$ the "matrix element" map $a \rightarrow(a u, v) \in C$ is continuous. By the Cauchy inequality, one sees that each of the matrix element functionals $(\cdot u, v)$ is in $A^{\prime}$, so the weak operator topology is coarser than the weak topology (properly coarser in the general infinite-dimensional case). When $\|u\|=1$, the functional $a \rightarrow(a u, u)$ is a positive functional on $B(X)$ and a state of every unital $B$-subalgebra $A$ of $B(X)$ (a point state), and the point state topology is the coarsest such that all of these functionals are continuous. The analog of Corollary 3 turns out to hold, for much the same reason. 
Proposition 4. The weak operator and point state topologies coincide on every $A \subset B(X)$. Indeed, every matrix element functional $f=(\cdot u, v)$ has the form

$$
(a u, v)=\sum\left\{i^{j} \alpha_{j}\left(a w_{j}, w_{j}\right): 0 \leqq j \leqq 3\right\} .
$$

Proof. First we write

$$
a=2^{-1}\left(a+a^{*}\right)+i(2 i)^{-1}\left(a-a^{*}\right)=h+i k
$$

so that $h^{*}=h, k^{*}=k$ in $B(X)$ (not necessarily in $A$ ). Then both $(u, v)_{h}=(h u, v)$ and $(u, v)_{k}=(k u, v)$ are sesquilinear forms, and by the polarization identity twice

$$
\begin{aligned}
& (h u, v)=4^{-1} \sum\left\{i^{j}\left(h\left(u+i^{j} v\right), u+i^{j} v\right): 0 \leqq j \leqq 3\right\}, \\
& (k u, v)=4^{-1} \sum\left\{i^{j}\left(k\left(u+i^{j} v\right), u+i^{j} v\right): 0 \leqq j \leqq 3\right\} .
\end{aligned}
$$

Hence

$$
(a u, v)=4^{-1} \sum\left\{i^{j}\left(a\left(u+i^{j} v\right), u+i^{j} v\right): 0 \leqq j \leqq 3\right\} .
$$

By taking $w_{j}=\left\|u+i^{j} v\right\|^{-1}\left(u+i^{j} v\right)$ and $\alpha_{j}=4^{-1}\left\|u+i^{j} v\right\|^{2}$, we obtain (1) from (4). Then since every matrix-element functional is in the complex span of the point states, the topologies induced by the two sets of functionals coincide, just as in Corollary 3.

This result also applies to homomorphisms $\rho$ of a locally compact group into $B(X)$ for $X$ a Hilbert space (alias group representations) to show that if $g \rightarrow(\rho(g) u, u)$ is continuous for all $u \in X$ with $\|u\|=1$, then $g \rightarrow(\rho(g) u, v)$ is continuous for all $u, v$ in $X$. Then by DeLeeuw-Glicksberg [4] again, $g \rightarrow \rho(g) u$ is continuous into $X$ for all $u \in X$.

Open Question. For which $B$-spaces $X$ and unital algebras $A \subset B(X)$ does Proposition 4 admit a reasonable extension?

This question was one of the primary motives behind the author's study of the easier questions affirmatively answered by Theorem 1(a) and Corollary 3. These results indicate at least that not all such phenomena depend upon $C^{*}$ structures and/or inner products, although the proof of Proposition 4 certainly relies upon these.

It is possible to specify the form that answers to the Open Question might take. The matrix element functionals are defined for a pair $\left(u, u^{\prime}\right)$ in $X \times X^{\prime}$ by $a \rightarrow u^{\prime}(a u)$; these define the weak-operator topology WO. The point states are defined by pairs $\left(u, u^{\prime}\right)$ such that $\|u\|=u^{\prime}(u)=\left\|u^{\prime}\right\|=1$ (so that $u^{\prime}$ is tangent to the unit $\|\cdot\|$-ball at $u \in X)$ by $a \rightarrow u^{\prime}(a u)$, and all point states are in $\Omega\left(A^{\prime}\right)$ (indeed, they are convex generators of $\Omega\left(A^{\prime}\right): \Omega\left(A^{\prime}\right)$ is the weak-* closed convex hull of the point states [7]). One can then define the point state topology $P S$ and ask when $P S=W O$ on all of $B(X)$ or when relativized to a subalgebra. It is not difficult to check that the Riesz representation theorem for the dual $X^{\prime}$ of a Hilbert space reduces this question to the one answered in Proposition 4 when $X$ is a Hilbert space. Clearly all topologies agree on finite-dimensional subalgebras such as $C I$ or those generated by $I$ and a 
projection or $I$ and an operator of finite rank. No nontrivial answers to the question are known, if the answer is sought in this form.

Answers to the question could take a second form, which may not be equivalent to the one just discussed when the unit ball in $X$ is nonsmooth. One takes a semiinner-product for $X$ (cf. Lumer [7]) and defines the [, ]-matrix element to be the functionals of the form $a \rightarrow[a u, v]$ for $u, v$ in $X$, while the [, ]-point states are of the form $a \rightarrow[a u, u]$ for $\|u\|=1$. Both of these sets of functionals can be proper subsets of the corresponding sets of functionals used to define $W O$ and $P S$, and may in some cases define topologies on $A$ different from both $W O$ and $P S$. Very little seems to be known about the possible interrelationships of these topologies.

\section{REFERENCES}

1. E. Berkson, Some characterizations of $C^{*}$-algebras, Illinois J. Math. 10 (1966), 1-8. MR 32 \#2922.

2. H. F. Bohnenblust and S. Karlin, Geometrical properties of the unit sphere of Banach algebras, Ann. of Math. (2) 62 (1955), 217-229. MR 17, 177.

3. F. F. Bonsall and J. Duncan, Numerical ranges of operators on normed spaces and of elements of normed algebras, London Math. Soc. Lecture Notes Ser. 2, Cambridge Univ. Press, London, 1971.

4. K. DeLeeuw and I. Glicksberg, The decomposition of certain group representations, J. Analyse Math. 15 (1965), 135-192.

5. J. Dixmier, Les $C^{*}$-algèbres et leurs représentations, Cahiers Scientifiques, fasc. 29 , Gauthier-Villars, Paris, 1964. MR 30 \#1404.

6. B. W. Glickfeld, $A$ metric characterization of $C(X)$ and its generalization to $C^{*}$-algebras, Illinois J. Math. 10 (1966), 547-556. MR 34 \#1865.

7. G. Lumer, Semi-inner-product spaces, Trans. Amer. Math. Soc. 100 (1961), 29-43. MR 24 \#A2860.

8. T. W. Palmer, Characterizations of $C^{*}$-algebras, Bull. Amer. Math. Soc. 74 (1968), 538-540. MR 36 \#5709.

9. H. H. Schaefer, Topological vector spaces, Macmillan, New York, 1966. MR 33 \#1689.

10. I. Vidav, Eine metrische Kennzeichnung der selbstadjungierten Operatoren, Math. Z. 66 (1956), 121-128. MR 18, 912.

University of Washington, Seattle, Washington 98105 